\title{
J-curve relationship between corrected QT interval and mortality in acute heart failure patients
}

\author{
Chan Soon Park ${ }^{1}$, Hyun-Jai Cho², Eue-Keun $\mathrm{Choi}^{2}$, Sang Eun Lee ${ }^{3}$, Min-Seok Kim³, Jae-Joong Kim³, \\ Jin-Oh Choi ${ }^{4}$, Eun-Seok Jeon ${ }^{4}$, Kyung-Kuk Hwang ${ }^{5}$, Shung Chull Chae ${ }^{6}$, Sang Hong Baek ${ }^{7}$, Seok-Min Kang ${ }^{8}$, \\ Byungsu Yoo ${ }^{9}$, Dong-Ju Choi ${ }^{10}$, Youngkeun $\mathrm{Ahn}^{11}$, Kye-Hoon Kim ${ }^{11}$, Myeong-Chan Cho ${ }^{5}$, Byung-Hee Oh ${ }^{12}$, \\ and Hae-Young Lee ${ }^{2}$
}

\author{
${ }^{1}$ Graduate School of Medical \\ Science and Engineering, Korea \\ Advanced Institute of Science and \\ Technology, Daejeon; ${ }^{2}$ Department \\ of Internal Medicine, Seoul National \\ University Hospital, Seoul; ${ }^{3}$ Division \\ of Cardiology, Department of \\ Internal Medicine, Asan Medical \\ Center, University of Ulsan College \\ of Medicine, Seoul; ${ }^{4}$ Department of \\ Internal Medicine, Sungkyunkwan \\ University College of Medicine, Seoul; \\ ${ }^{5}$ Department of Internal Medicine, \\ Chungbuk National University College \\ of Medicine, Cheongju; ${ }^{6}$ Department \\ of Internal Medicine, School of \\ Medicine, Kyungpook National \\ University, Daegu; ${ }^{7}$ Department \\ of Internal Medicine, College of \\ Medicine, The Catholic University of \\ Korea, Seoul; ${ }^{8}$ Department of Internal \\ Medicine, Yonsei University College \\ of Medicine, Seoul; ${ }^{9}$ Department of \\ Internal Medicine, Yonsei University \\ Wonju College of Medicine, Wonju; \\ ${ }^{10}$ Cardiovascular Center, Division of \\ Cardiology, Seoul National University \\ Bundang Hospital, Seongnam; ${ }^{11}$ Heart \\ Research Center, Chonnam National \\ University, Gwangju; ${ }^{12}$ Department of \\ Internal Medicine, Mediplex Sejong \\ Hospital, Incheon, Korea
}

Background/Aims: This study investigated the prognostic power of corrected QT (QTc) interval in patients with acute heart failure (AHF) according to sex.

Methods: We analyzed multicenter Korean Acute Heart Failure registry with patients with AHF admitted from 2011 to 2014. Among them, we analyzed 4,990 patients who were followed up to 5 years. Regarding QTc interval based on 12 lead electrocardiogram, patients were classified into quartiles according to sex.

Results: During follow-up with median 43.7 months, 2,243 (44.9\%) patients died. The relationship between corrected QT interval and all-cause mortality followed a J-curve relationship. In Kaplan-Meier analysis, both sex had lowest mortality in the second QTc quartile. There were significant prognostic differences between the second and the fourth quartiles in male $(\log$-rank $p=0.002)$, but not in female $(\log$-rank $p=0.338)$. After adjusting covariates, the third (hazard ratio $[\mathrm{HR}], 1.185$; 95\% confidence interval [CI], 1.001 to $1.404 ; p=0.049)$ and the fourth $(\mathrm{HR}, 1.404$; $95 \%$ CI, 1.091 to $1.535 ; p=0.003$ ) quartiles demonstrated increased risk of mortality compared to the second quartile in male. In female, however, there was no significant difference across quartiles. QTc interval was associated with 5-year all-cause mortality in J-shape with nadir of 440 to $450 \mathrm{~ms}$ in male and 470 to $480 \mathrm{~ms}$ in female.

Conclusions: QTc interval was an independent predictor of overall death in male, but its significance decreased in female. The relationship between QTc interval and all-cause mortality was J-shaped in both sex.

Keywords: Heart failure; QTc interval; Sex; Mortality; Prognosis

Correspondence to Hae Young Lee, M.D.

Department of Internal Medicine, Seoul National University College of Medicine, 101 Daehak-ro, Jongno-gu, Seoul 03080, Korea

Tel: +82-2-2072-0698, Fax: +82-2-3674-0805, E-mail: hylee612@snu.ac.kr

https://orcid.org/0000-0002-9521-4102 


\section{INTRODUCTION}

Despite the advances in heart failure (HF) management, the associated mortality rate still remains substantially high [1-3]. When patients are hospitalized with acutely decompensated HF, they demonstrate high rates of readmission and mortality [4-7]. Therefore, various studies have been conducted to improve the precision of risk stratification in patients with acute $\mathrm{HF}[8,9]$. Guidelines for the diagnosis and treatment of acute and chronic HF recommend an evaluation with 12-lead electrocardiogram (ECG) for all patients presenting with symptoms and signs of $\mathrm{HF}[10,11]$. Clinical information obtained from ECG, such as heart rhythm, heart rate, QRS morphology, and QT interval, assists in diagnosing cardiac abnormalities, making treatment decision, and predicting prognosis.

QT interval reflects the duration of ventricular depolarization and repolarization. Corrected QT (QTc) interval is adjusted according to heart rate, which helps to identify patients with increased risk of ventricular arrhythmia [12]. Left ventricular dilatation and female sex have been reported to be associated with QTc prolongation $[13,14]$. Owing to their association with electrical instability and arrhythmogenesis, prolonged QT and QTc interval have been considered to be useful prognosticators $[15,16]$. These intervals have shown prognostic value, possibly with a J-shaped association, in healthy individuals as well as in those with risk factors [17-21]. Furthermore, these intervals have also been reported to have predictive value in patients with chronic $\operatorname{HF}[22,23]$.

On the contrary, a previous small and short cohort study in patients with acute HF failed to demonstrate significant association between prolonged QTc and mortality [24]. However, this study only performed binary risk stratification (QTc $\geq 440 \mathrm{~ms}$ or less), and did not consider QTc difference according to sex.

In this study, we hypothesized that QTc interval has useful prognostic value in patients with acute $\mathrm{HF}$, and its association would be different according to sex. We categorized the patients in quartiles according to QTc interval and sex, and then investigated the prognostic value of QTc interval using 5-year follow-up data from Korean acute HF (KorAHF) registry.

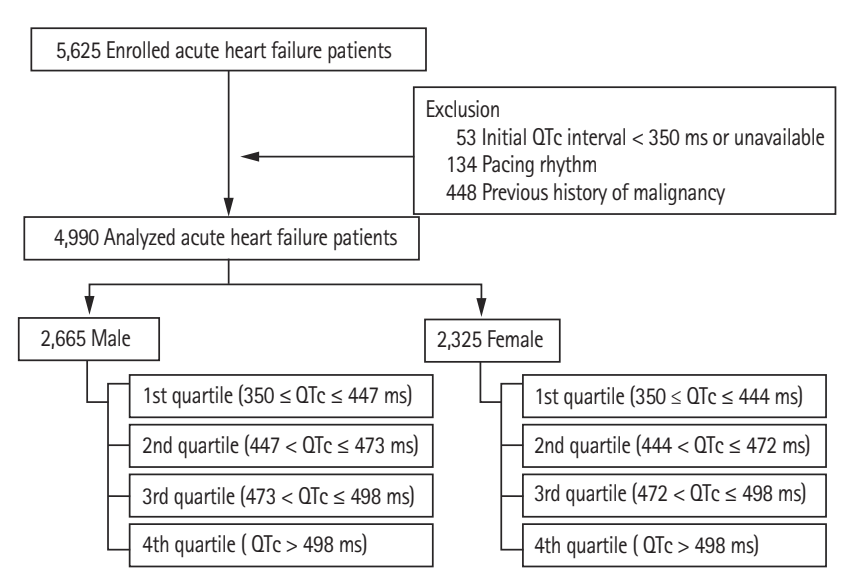

Figure 1. Scheme of the study. Scheme of this study is presented. QTc, corrected QT.

\section{METHODS}

The design of the KorAHF registry has been published elsewhere $[2,3]$. In brief, this multicenter cohort registry was designed to include consecutive patients admitted to the hospital with acute HF syndrome. Of the 5,625 patients recruited between January 2011 and February 2014, patients with QTc interval < $350 \mathrm{~ms}(\mathrm{n}=53)$, pacing rhythm ( $\mathrm{n}=134)$, and a previously diagnosed malignant neoplasm $(n=448)$ were excluded. Patients with the history of malignancy were excluded because their mortality was significantly different from that of patients without the history of malignancy. Final 4,990 patients were included in the study. A schematic flowchart of this study is presented in Fig. 1. The Institutional Review Board of Seoul National University Hospital approved the study protocol (IRB No.: 2004-166-1119). Written informed consent by the patients was waived due to a retrospective nature of our study. The investigation conformed to the principles outlined in the Declaration of Helsinki.

We recorded demographics, past medical history, social history, clinical presentation, and physical examination. Further, leukocytes, hemoglobin, blood urea nitrogen, creatinine, sodium, and potassium levels were measured and recorded at admission. Blood sampling and tests were conducted by laboratories at each center certified by the Korean Association of Quality Assurance for Clinical Laboratories. Transthoracic echocardiography was performed by a skilled clinician upon admission. Data were entered into registry database through 
a web-based case report form filled by the attending physician. Patients underwent standard 12-lead ECG evaluation upon admission. For this study we used clinical QTc data obtained from these initial 12-lead ECG recordings, which were performed in real-world clinics. Patients were classified into quartiles according to sex; men were classified into 1 st ( $350 \leq \mathrm{QTc} \leq 447 \mathrm{~ms})$, and ( $447<$ QTc $\leq 473 \mathrm{~ms})$, 3rd $(473<$ QTc $\leq 498 \mathrm{~ms})$, and 4 th (QTc > $498 \mathrm{~ms}$ ) quartiles and women were stratified into 1st ( $350 \leq \mathrm{QTc} \leq 444 \mathrm{~ms})$, 2nd ( $444<\mathrm{QTc} \leq 472 \mathrm{~ms})$, 3rd $(472<\mathrm{QTc} \leq 498 \mathrm{~ms})$, and 4 th $(\mathrm{QTc}>498 \mathrm{~ms})$ quartiles.

Patients were followed up for 1, 3, 6, 12, 24, 36, 48, and 60 months. All-cause mortality was used as a primary outcome variable in this study. Rate of any death or heart transplantation was the secondary outcome variable. Mortality data of patients who were lost to follow-up were collected from the governmental statistics office in South Korea. The primary endpoint was 5-year all-cause mortality, and we also analyzed 30-day mortality. In addition, we analyzed the 5-year and 30-day composite outcomes of all-cause mortality and hospitalization due to heart failure.

\section{Statistical analysis}

Data were described as numbers and frequencies for categorical variables, and as mean \pm standard deviation or median with interquartile range for continuous variables. For comparison between groups, the chi-square test or Fisher's exact test was used for categorical variables and the unpaired Student's $t$ test was used for continuous variables. One-way analysis of variance and Scheffe's post hoc test were used to analyze differences between continuous variables among $>2$ groups. The chronological trend of outcomes was expressed as Kaplan-Meier estimates and compared according to the QTc interval. The log-rank test was used to analyze the significant differences in clinical outcomes. A multivariable Cox proportional-hazards regression model was used to find independent predictors of clinical outcomes. The associations between QTc interval and all-cause mortality were investigated using multivariable fractional polynomials (MFP). Two-sided $p<0.05$ was considered statistically significant. The IBM SPSS Statistics version 23 (IBM Co., Armonk, NY, USA) and STATA software version 12 (Stata Co., College Station, TX, USA) were used to perform statistical tests.

\section{RESULTS}

\section{Baseline characteristics of study population}

The baseline characteristics of patients according to sex and QTc quartiles are described in Tables 1 and 2. Briefly, the median follow-up period was 43.7 months and the mean age was 68.3 years, $53.4 \%$ of the subjects were males, $59.5 \%$ had hypertension, $36.0 \%$ had diabetes mellitus, and $28.8 \%$ had ischemic heart disease. In the study population, $42.4 \%$ of the subjects had been previously diagnosed with heart failure. There were no significant differences in age, previous history of hypertension, diabetes mellitus, heart failure, ischemic heart disease, chronic obstructive lung disease, chronic kidney disease, and cerebrovascular accident across the QTc quartiles in both sex. Baseline characteristics according to sex are demonstrated in Supplementary Table 1.

Though previous report defined normal QTc group as QTc < $440 \mathrm{~ms}$ in patients with acute HF [24], the upper limit of 1st quartile surpass $440 \mathrm{~ms}$ in both male and female as shown in Tables 1 and 2. Left ventricular end diastolic diameter was significantly associated with QTc interval $(r=0.133, p<0.001$ for male and $r=0.181, p<$ 0.001 for female). Age did not show significant correlation with QTc interval $(r=-0.006, p=0.752$ for male and $r=-0.029, p=0.160$ for female) in both groups. LVEF was negatively associated with QTc interval in both sexes $(r$ $=-0.204, p<0.001$ for males and $r=-0.225, p<0.001$ for females).

\section{Clinical outcomes according to QTc quartiles during follow-up}

During the 5-year follow-up period, 2,243 patients (44.9\%) died, of which 1,185 (52.8\%) were male. Among them, 268 patients died during first 30 days. Subjects who died had more adverse characteristics, including older age, higher prevalence of previous hypertension, diabetes mellitus, heart failure, ischemic heart disease, valvular heart disease, chronic lung disease, chronic kidney disease, and cerebrovascular accident, than the survivors. Deceased subjects had larger left ventricular end diastolic diameter; however, there was no significant difference between the left ventricular ejection fractions of the living and deceased subjects (Supplementary Table 2).

Kaplan-Meier analysis was performed to demonstrate the clinical outcome of patients according to the QTC 
Table 1. Baseline characteristics of male patients according to corrected QT Interval (total $\mathbf{n}=\mathbf{2 , 6 6 5}$ )

\begin{tabular}{|c|c|c|c|c|c|}
\hline Characteristic & $\begin{array}{c}\text { 1st quartile } \\
\begin{array}{c}(350 \leq \mathrm{QTc} \leq 447 \mathrm{~ms}) \\
(\mathrm{n}=685)\end{array}\end{array}$ & $\begin{array}{c}\text { 2nd quartile } \\
(447<\text { QTc } \leq 473 \mathrm{~ms}) \\
(\mathrm{n}=666)\end{array}$ & $\begin{array}{c}\text { 3rd quartile } \\
(473<\text { QTc } \leq 498 \mathrm{~ms}) \\
(\mathrm{n}=649)\end{array}$ & $\begin{array}{c}\text { 4th quartile } \\
(\mathrm{QTc}>498 \mathrm{~ms}) \\
(\mathrm{n}=664)\end{array}$ & $p$ value \\
\hline \multicolumn{6}{|l|}{ Demographic data } \\
\hline Age, yr & $65.9 \pm 15.1$ & $64.7 \pm 15.2$ & $64.7 \pm 65.8$ & $65.8 \pm 14.8$ & 0.249 \\
\hline $\mathrm{BMI}, \mathrm{kg} / \mathrm{m}^{2}$ & $23.8 \pm 3.8$ & $23.6 \pm 3 \cdot 5$ & $23.8 \pm 4.1$ & $23.3 \pm 3.6$ & 0.078 \\
\hline \multicolumn{6}{|l|}{ Past medical history } \\
\hline Hypertension & $396(57.8)$ & $375(56.3)$ & $362(55.8)$ & $352(53.0)$ & 0.350 \\
\hline Diabetes mellitus & $257(37.5)$ & $242(36.3)$ & $235(36.2)$ & $231(34.8)$ & 0.779 \\
\hline Heart failure & $269(39 \cdot 3)$ & $270(40.5)$ & $260(40.1)$ & $293(44.1)$ & 0.281 \\
\hline Ischemic heart disease & $209(30.5)$ & $225(33.8)$ & $208(32.0)$ & $211(31.9)$ & 0.631 \\
\hline Valvular heart disease & $90(13.1)$ & $71(10.7)$ & $73(11.2)$ & $74(11.1)$ & 0.499 \\
\hline COLD & $109(15 \cdot 9)$ & $84(12.6)$ & $73(11.2)$ & $86(13.0)$ & 0.080 \\
\hline CKD & $113(16.5)$ & $99(14.9)$ & $117(18.0)$ & $85(12.8)$ & 0.056 \\
\hline $\begin{array}{l}\text { Cerebrovascular } \\
\text { accident }\end{array}$ & $105(15 \cdot 3)$ & $115(17 \cdot 3)$ & $93(14.4)$ & $116(17.5)$ & 0.339 \\
\hline \multicolumn{6}{|l|}{ Social history } \\
\hline Smoking history & & & & & 0.062 \\
\hline Current smoker & $194(28.3)$ & $236(35 \cdot 4)$ & $192(29.6)$ & $204(30.7)$ & \\
\hline Ex-smoker & $251(36.6)$ & $234(35 \cdot 1)$ & $222(34.2)$ & $235(35 \cdot 4)$ & \\
\hline Never smoker & $240(35.0)$ & $196(29.4)$ & $235(36.2)$ & $225(33.9)$ & \\
\hline Alcohol history & & & & & 0.013 \\
\hline Heavy drinker & $62(9.1)$ & $81(12.2)$ & $84(12.9)$ & $100(15.1)$ & \\
\hline Social drinker & $350(51.1)$ & $315(47 \cdot 3)$ & $328(50.5)$ & $295(44.4)$ & \\
\hline Never drinker & $273(39.9)$ & $270(40.5)$ & $237(36.5)$ & $269(40.5)$ & \\
\hline \multicolumn{6}{|l|}{ Major ECG abnormalities } \\
\hline Atrial fibrillation & $207(30.2)$ & $165(24.8)$ & $165(25 \cdot 4)$ & $165(24.9)$ & 0.067 \\
\hline $\begin{array}{l}\text { Right bundle branch } \\
\text { block }\end{array}$ & $19(2.8)$ & $26(3.9)$ & $55(8.5)$ & $122(18.4)$ & $<0.001$ \\
\hline $\begin{array}{l}\text { Left bundle branch } \\
\text { block }\end{array}$ & $6(0.9)$ & $13(2.0)$ & $17(2.6)$ & $84(12.5)$ & $<0.001$ \\
\hline NYHA functional class & & & & & 0.482 \\
\hline Stage II & $115(16.8)$ & $123(18.5)$ & $98(15 \cdot 1)$ & $101(15.2)$ & \\
\hline Stage III & $247(36.1)$ & $248(37.2)$ & $247(38.1)$ & $236(35 \cdot 5)$ & \\
\hline Stage IV & $323(47.2)$ & $295(44 \cdot 3)$ & $304(46.8)$ & $327(49.2)$ & \\
\hline \multicolumn{6}{|l|}{ Physical exam } \\
\hline SBP, mmHg & $129.8 \pm 29.6$ & $131.1 \pm 31.5$ & $132.37 \pm 30.8$ & $127.0 \pm 30.5$ & 0.011 \\
\hline $\mathrm{DBP}, \mathrm{mmHg}$ & $76.5 \pm 18.5$ & $79.5 \pm 19.1$ & $80.7 \pm 19.2$ & $79.1 \pm 20.0$ & 0.001 \\
\hline HR, beats/min & $87.9 \pm 26.0$ & $91.4 \pm 24.3$ & $94 \cdot 6 \pm 25 \cdot 4$ & $95.2 \pm 26.8$ & $<0.001$ \\
\hline \multicolumn{6}{|l|}{ Laboratory exam } \\
\hline Hemoglobin, mg/dL & $14.6 \pm 2.3$ & $14.5 \pm 2.4$ & $14.7 \pm 2.0$ & $14.6 \pm 1.9$ & 0.360 \\
\hline Sodium, mmol/L & $137 \cdot 6 \pm 4.7$ & $137 \cdot 9 \pm 4 \cdot 3$ & $137 \cdot 6 \pm 4 \cdot 5$ & $137.0 \pm 4.9$ & 0.004 \\
\hline Potassium, mmol/L & $4.4 \pm 0.7$ & $4.4 \pm 0.6$ & $4.4 \pm 0.6$ & $4.4 \pm 0.7$ & 0.618 \\
\hline $\mathrm{BUN}, \mathrm{mg} / \mathrm{dL}$ & $26.7 \pm 17.4$ & $25.6 \pm 15.9$ & $26.8 \pm 15.8$ & $27.1 \pm 16.5$ & 0.336 \\
\hline
\end{tabular}


Table 1. Continued

\begin{tabular}{|c|c|c|c|c|c|}
\hline Characteristic & $\begin{array}{c}\text { 1st quartile } \\
(350 \leq \mathrm{QTc} \leq 447 \mathrm{~ms}) \\
(\mathrm{n}=685)\end{array}$ & $\begin{array}{c}\text { 2nd quartile } \\
(447<\text { QTc } \leq 473 \mathrm{~ms}) \\
(\mathrm{n}=666)\end{array}$ & $\begin{array}{c}\text { 3rd quartile } \\
(473<\mathrm{QTc} \leq 498 \mathrm{~ms}) \\
(\mathrm{n}=649)\end{array}$ & $\begin{array}{c}\text { 4th quartile } \\
\text { (QTc > } 498 \mathrm{~ms}) \\
(\mathrm{n}=664)\end{array}$ & $p$ value \\
\hline Creatinine, mg/dL & $1.7 \pm 1.9$ & $1.6 \pm 1.4$ & $1.7 \pm 1.7$ & $1.7 \pm 1.7$ & 0.593 \\
\hline $\mathrm{BNP}, \mathrm{pg} / \mathrm{mL}$ & $\begin{array}{c}667.4 \\
(283.5-1,487.5)\end{array}$ & $\begin{array}{c}753.0 \\
(424.8-1,436.5)\end{array}$ & $\begin{array}{c}902.1 \\
(478.0-1,627.5)\end{array}$ & $\begin{array}{c}1,124 \cdot 4 \\
(579 \cdot 5-2,441.5)\end{array}$ & $<0.001$ \\
\hline NT-proBNP, pg/mL & $\begin{array}{c}3,558.0 \\
(1,304 \cdot 9-9,477.0)\end{array}$ & $\begin{array}{c}4,460.0 \\
(2,104 \cdot 5-1,0314 \cdot 5)\end{array}$ & $\begin{array}{c}4,502.0 \\
(2,078.8-9,860.0)\end{array}$ & $\begin{array}{c}4,641.2 \\
(2,441.5-1,1871.0)\end{array}$ & 0.074 \\
\hline \multicolumn{6}{|l|}{ Medication at admission } \\
\hline Beta-blockers & $203(29.6)$ & $181(27.2)$ & $158(24 \cdot 3)$ & $174(26.2)$ & 0.181 \\
\hline $\begin{array}{l}\text { Renin-angiotensin- } \\
\text { system inhibitors }\end{array}$ & $270(39.4)$ & $256(38.4)$ & $225(34.7)$ & $259(39.0)$ & 0.270 \\
\hline $\begin{array}{l}\text { Mineralocorticoid } \\
\text { receptor blockers }\end{array}$ & $124(18.1)$ & $111(16.7)$ & $103(15 \cdot 9)$ & $121(18.2)$ & 0.617 \\
\hline \multicolumn{6}{|l|}{ Medication at discharge } \\
\hline Beta-blockers & $317(46.2)$ & $354(53 \cdot 2)$ & $317(48.8)$ & $341(51.5)$ & 0.057 \\
\hline $\begin{array}{l}\text { Renin-angiotensin- } \\
\text { system inhibitors }\end{array}$ & $441(64 \cdot 3)$ & $470(70.6)$ & $442(68.1)$ & $467(70.3)$ & 0.047 \\
\hline $\begin{array}{l}\text { Mineralocorticoid } \\
\text { receptor blockers }\end{array}$ & $262(38.2)$ & $294(44.1)$ & $313(48.2)$ & $318(47 \cdot 9)$ & $<0.001$ \\
\hline \multicolumn{6}{|c|}{ Echocardiographic findings } \\
\hline $\begin{array}{l}\text { Left ventricular end } \\
\text { diastolic diameter, } \\
\text { mm }\end{array}$ & $57.9 \pm 10.0$ & $60.6 \pm 9.5$ & $60.9 \pm 9.9$ & $61.2 \pm 9.9$ & $<0.001$ \\
\hline Ejection fraction, \% & $39.2 \pm 15.5$ & $34 \cdot 6 \pm 14 \cdot 4$ & $33 \cdot 4 \pm 14.1$ & $30.8 \pm 12.9$ & $<0.001$ \\
\hline $\begin{array}{l}\text { Heart failure with } \\
\text { reduced ejection } \\
\text { fraction }(<50 \%)\end{array}$ & $485(73.8)$ & $546(84 \cdot 5)$ & $536(85.8)$ & $583(91.0)$ & $<0.001$ \\
\hline
\end{tabular}

Values are presented as mean $\pm \mathrm{SD}$, number (\%), or median (range).

QTc, corrected QT; BMI, body mass index; COLD, chronic obstructive lung disease; CKD, chronic kidney disease; ECG, electrocardiogram; NYHA, New York Heart association; SBP, systolic blood pressure; DBP, diastolic blood pressure; HR, heart rate; BUN, blood urea nitrogen; BNP, B-type natriuretic peptide; NT-proBNP, N-terminal proBNP.

quartiles in both sex. In male, 2nd quartile not only showed highest survival rates but also showed significantly superior prognosis compared to 4 th quartile which had lowest survival rates (log-rank $p=0.002$ ) (Fig. 2A). On the contrary, there was no significant difference between 2 nd and 4 th quartiles (log-rank $p=0.338)$ in female (Fig. 2B). This tendency was also observed in the 30-day mortality, as demonstrated in Fig. 3. When composite outcomes of mortality and hospitalization due to heart failure were analyzed, only the 4 th quartile of male patients showed increased risk at 5 years, with no prog- nostic difference across quartiles seen in female patients (Supplementary Fig. 1).

\section{Different prognostic impact of QTc quartiles ac- cording to sex}

The results of Cox-proportional regression analysis for 5-year mortality were shown in Table 3. The and quartile was defined as a reference group, as it had lowest risk in Kaplan-Meier analysis in both sex. Univariate analysis showed increased risk of 4 th quartile in male (hazard ratio [HR], 1.289; 95\% confidence interval [CI], 1.095 to 
Table 2. Baseline characteristics of female patients according to Corrected QT Interval (total $\mathbf{n}=\mathbf{2 , 3 2 5}$ )

\begin{tabular}{|c|c|c|c|c|c|}
\hline Characteristic & $\begin{array}{c}\text { 1st quartile } \\
(350 \leq \mathrm{QTc} \leq 444 \mathrm{~ms}) \\
(\mathrm{n}=599)\end{array}$ & $\begin{array}{c}\text { 2nd quartile } \\
(444<\mathrm{QTc} \leq 472 \mathrm{~ms}) \\
(\mathrm{n}=581)\end{array}$ & $\begin{array}{c}\text { 3rd quartile } \\
(472<\mathrm{QTc} \leq 498 \mathrm{~ms}) \\
(\mathrm{n}=573)\end{array}$ & $\begin{array}{c}\text { 4th quartile } \\
(\mathrm{QTc}>498 \mathrm{~ms}) \\
(\mathrm{n}=572)\end{array}$ & $p$ value \\
\hline \multicolumn{6}{|l|}{ Demographic data } \\
\hline Age, yr & $72.1 \pm 14.5$ & $71.4 \pm 13.5$ & $72.1 \pm 13.4$ & $71.6 \pm 13.8$ & 0.750 \\
\hline BMI, $\mathrm{kg} / \mathrm{m}^{2}$ & $23.0 \pm 4.2$ & $23.4 \pm 4.0$ & $23.0 \pm 3.8$ & $22.8 \pm 4.2$ & 0.038 \\
\hline \multicolumn{6}{|l|}{ Past medical history } \\
\hline Hypertension & $368(61.4)$ & $387(66.6)$ & $380(66.3)$ & $348(60.8)$ & 0.066 \\
\hline Diabetes mellitus & $209(34.9)$ & $216(37.2)$ & $212(37.0)$ & $194(33.9)$ & 0.586 \\
\hline Heart failure & $260(43 \cdot 4)$ & $253(43.5)$ & $256(44 \cdot 7)$ & $252(44.1)$ & 0.972 \\
\hline Ischemic heart disease & $139(23.2)$ & $152(26.2)$ & $163(28.4)$ & $128(22.4)$ & 0.067 \\
\hline Valvular heart disease & $135(22.5)$ & $95(16.4)$ & $96(16.8)$ & $94(16.5)$ & 0.012 \\
\hline COLD & $53(8.8)$ & $50(8.6)$ & $51(8.9)$ & $43(7 \cdot 5)$ & 0.818 \\
\hline CKD & $58(9.7)$ & $81(13.9)$ & $78(13.6)$ & $67(11.7)$ & 0.095 \\
\hline Cerebrovascular accident & $95(15 \cdot 9)$ & $77(13 \cdot 3)$ & $81(14.1)$ & $89(15.6)$ & 0.553 \\
\hline \multicolumn{6}{|l|}{ Social history } \\
\hline Smoking history & & & & & 0.534 \\
\hline Current smoker & $23(3.8)$ & $25(4 \cdot 3)$ & $32(5 \cdot 6)$ & $30(5.2)$ & \\
\hline Ex-smoker & $20(3 \cdot 3)$ & $16(2.8)$ & $24(4.2)$ & $16(2.8)$ & \\
\hline Never smoker & $556(92.8)$ & $540(92.9)$ & $517(90.2)$ & $526(92.0)$ & \\
\hline Alcohol history & & & & & 0.451 \\
\hline Heavy drinker & $5(0.8)$ & $5(0.9)$ & $5(0.9)$ & $7(1.2)$ & \\
\hline Social drinker & $79(13.2)$ & $80(13.8)$ & $76(13 \cdot 3)$ & $56(9.8)$ & \\
\hline Never drinker & $515(86.0)$ & $496(85.4)$ & $492(85.9)$ & $509(89.0)$ & \\
\hline \multicolumn{6}{|l|}{ Major ECG abnormalities } \\
\hline Atrial fibrillation & $207(34.6)$ & $184(31.7)$ & $150(26.2)$ & $148(25.9)$ & 0.002 \\
\hline $\begin{array}{l}\text { Right bundle } \\
\text { branch block }\end{array}$ & $11(1.8)$ & $24(4 \cdot 1)$ & $29(5.1)$ & $74(12.9)$ & $<0.001$ \\
\hline Left bundle branch block & $5(0.8)$ & $9(1.5)$ & $24(4.2)$ & $112(19.6)$ & $<0.001$ \\
\hline NYHA functional class & & & & & 0.747 \\
\hline Stage II & $87(14.5)$ & $86(14.8)$ & $79(13.8)$ & $69(12.1)$ & \\
\hline Stage III & $224(37.4)$ & $204(35.1)$ & $203(35.4)$ & $204(35 \cdot 7)$ & \\
\hline Stage IV & $288(48.1)$ & $291(50.1)$ & $291(50.8)$ & $299(52.3)$ & \\
\hline \multicolumn{6}{|l|}{ Physical exam } \\
\hline SBP, mmHg & $133.5 \pm 30.4$ & $136.4 \pm 31.0$ & $134.6 \pm 28.3$ & $132.5 \pm 29.9$ & 0.151 \\
\hline $\mathrm{DBP}, \mathrm{mmHg}$ & $78.2 \pm 18.2$ & $80.1 \pm 19.4$ & $80.0 \pm 18.2$ & $79.1 \pm 17.7$ & 0.238 \\
\hline HR, beats/min & $91.7 \pm 27.8$ & $94.1 \pm 25 \cdot 3$ & $93.8 \pm 24.6$ & $94.1 \pm 24 \cdot 4$ & 0.312 \\
\hline \multicolumn{6}{|l|}{ Laboratory exam } \\
\hline Hemoglobin, mg/dL & $14.8 \pm 2.2$ & $14.6 \pm 1.8$ & $14.6 \pm 1.7$ & $14.7 \pm 1.9$ & 0.386 \\
\hline Sodium, mmol/L & $137.2 \pm 5 \cdot 3$ & $137 \cdot 9 \pm 4 \cdot 5$ & $137.9 \pm 5.0$ & $137 \cdot 5 \pm 5 \cdot 0$ & 0.068 \\
\hline Potassium, mmol/L & $4.4 \pm 0.7$ & $4.4 \pm 0.7$ & $4.3 \pm 0.7$ & $4.2 \pm 0.7$ & $<0.001$ \\
\hline BUN, mg/dL & $25.0 \pm 16.6$ & $25.1 \pm 15.7$ & $25.4 \pm 14.7$ & $26.0 \pm 17.8$ & 0.722 \\
\hline
\end{tabular}


Table 2. Continued

\begin{tabular}{|c|c|c|c|c|c|}
\hline Characteristic & $\begin{array}{c}\text { 1st quartile } \\
(350 \leq \mathrm{QTc} \leq 444 \mathrm{~ms}) \\
(\mathrm{n}=599)\end{array}$ & $\begin{array}{c}\text { 2nd quartile } \\
(444<\text { QTc } \leq 472 \mathrm{~ms}) \\
(\mathrm{n}=58 \mathrm{1})\end{array}$ & $\begin{array}{c}\text { 3rd quartile } \\
(472<\text { QTc } \leq 498 \mathrm{~ms}) \\
(\mathrm{n}=573)\end{array}$ & $\begin{array}{c}\text { 4th quartile } \\
\text { (QTc > } 498 \mathrm{~ms}) \\
(\mathrm{n}=572)\end{array}$ & $p$ value \\
\hline Creatinine, mg/dL & $1.2 \pm 0.9$ & $1.3 \pm 1.2$ & $1.3 \pm 1.5$ & $1.3 \pm 1.2$ & 0.338 \\
\hline $\mathrm{BNP}, \mathrm{pg} / \mathrm{mL}$ & $\begin{array}{c}697.5 \\
(336.3-1,427.1)\end{array}$ & $\begin{array}{c}905.5 \\
(505.6-1,749.0)\end{array}$ & $\begin{array}{c}1018.2 \\
(534.1-1,744.5)\end{array}$ & $\begin{array}{c}1152.0 \\
(607.0-2,372.1)\end{array}$ & $<0.001$ \\
\hline NT-proBNP, pg/mL & $\begin{array}{c}5,068.9 \\
(1,852.4-12,957 \cdot 3)\end{array}$ & $\begin{array}{c}5,185 \cdot 5 \\
(2,317.8-12,534 \cdot 5)\end{array}$ & $\begin{array}{c}5,435 \cdot 0 \\
(2,377 \cdot 3-15,115 \cdot 3)\end{array}$ & $\begin{array}{c}6302.0 \\
(2,938.0-14,814.0)\end{array}$ & 0.162 \\
\hline \multicolumn{6}{|l|}{ Medication at admission } \\
\hline Beta-blockers & $170(28.4)$ & $200(34.4)$ & $186(325)$ & $148(25 \cdot 9)$ & 0.006 \\
\hline $\begin{array}{l}\text { Renin-angiotensin- } \\
\text { system inhibitors }\end{array}$ & $239(39.9)$ & $225(38.7)$ & $228(39.8)$ & $188(32.9)$ & 0.042 \\
\hline $\begin{array}{l}\text { Mineralocorticoid } \\
\text { receptor blockers }\end{array}$ & $145(24.2)$ & $103(17.7)$ & $97(16.9)$ & $106(18.5)$ & 0.006 \\
\hline \multicolumn{6}{|l|}{ Medication at discharge } \\
\hline Beta-blockers & $249(41.6)$ & $283(48.7)$ & $274(47.8)$ & $284(49 \cdot 7)$ & 0.023 \\
\hline $\begin{array}{l}\text { Renin-angiotensin- } \\
\text { system inhibitors }\end{array}$ & $363(60.6)$ & $374(64 \cdot 4)$ & $383(68.6)$ & $390(68.2)$ & 0.035 \\
\hline $\begin{array}{l}\text { Mineralocorticoid } \\
\text { receptor blockers }\end{array}$ & $264(44.1)$ & $246(42.3)$ & $270(47.1)$ & $286(50.0)$ & 0.046 \\
\hline \multicolumn{6}{|l|}{ Echocardiographic findings } \\
\hline $\begin{array}{l}\text { Left ventricular end } \\
\text { diastolic diameter, } \mathrm{mm}\end{array}$ & $52.0 \pm 8.7$ & $53 \cdot 3 \pm 9.1$ & $55.2 \pm 8.9$ & $57.1 \pm 9.9$ & $<0.001$ \\
\hline Ejection fraction, \% & $45 \cdot 3 \pm 15 \cdot 9$ & $44 \cdot 0 \pm 15 \cdot 5$ & $39.7 \pm 15.3$ & $36.4 \pm 14.8$ & $<0.001$ \\
\hline $\begin{array}{l}\text { Heart failure with } \\
\text { reduced ejection } \\
\text { fraction }(<50 \%)\end{array}$ & $334(57 \cdot 7)$ & $363(64.5)$ & $387(71.1)$ & $447(80.5)$ & $<0.001$ \\
\hline
\end{tabular}

Values are presented as mean $\pm \mathrm{SD}$, number (\%), or median (interquartile range).

QTc, corrected QT; BMI, body mass index; COLD, chronic obstructive lung disease; CKD, chronic kidney disease; ECG, electrocardiogram; NYHA, New York Heart association; SBP, systolic blood pressure; DBP, diastolic blood pressure; HR, heart rate; BUN, blood urea nitrogen; BNP, B-type natriuretic peptide; NT-proBNP, N-terminal proBNP .

1.516; $p=0.002$ ). In female, no statistical significance was observed across the quartiles.

We then evaluated the multivariate analysis, adjusted for atrial fibrillation and right or left bundle branch block (model 1), and for comprehensive clinical data including age, body mass index, previous history of hypertension, diabetes mellitus, heart failure, ischemic heart disease, valvular heart disease, chronic obstructive lung disease, chronic renal failure, cerebrovascular accident, atrial fibrillation, beta-blocker medication at discharge, and right or left bundle branch block (model 2). With both models, 4 th quartile in male had worse prognosis than reference group $(\mathrm{HR}, 1.252 ; 95 \% \mathrm{CI}, 1.058$ to $1.481 ; p=$
0.009 for model 1 and HR 1.404; 95\% CI, 1.091 to 1.535; $p=$ 0.003 for model 2). In addition, 3 th quartile in male also showed detrimental prognosis (HR, 1.185; 95\% CI, 1.001 to $1.404 ; p=0.049)$ in model 2 . However, there was no significant prognostic difference across QTc quartiles of female. The Cox model with MFP showed a J-shaped association in both male and female (Fig. 4). Male had nadir in about 440 to $450 \mathrm{~ms}$, female in about 470 to 480 ms with less steeper slope. When multivariate analyses were performed for 30-day mortality, there was no significant difference across quartiles in both male and female (Table 4). 


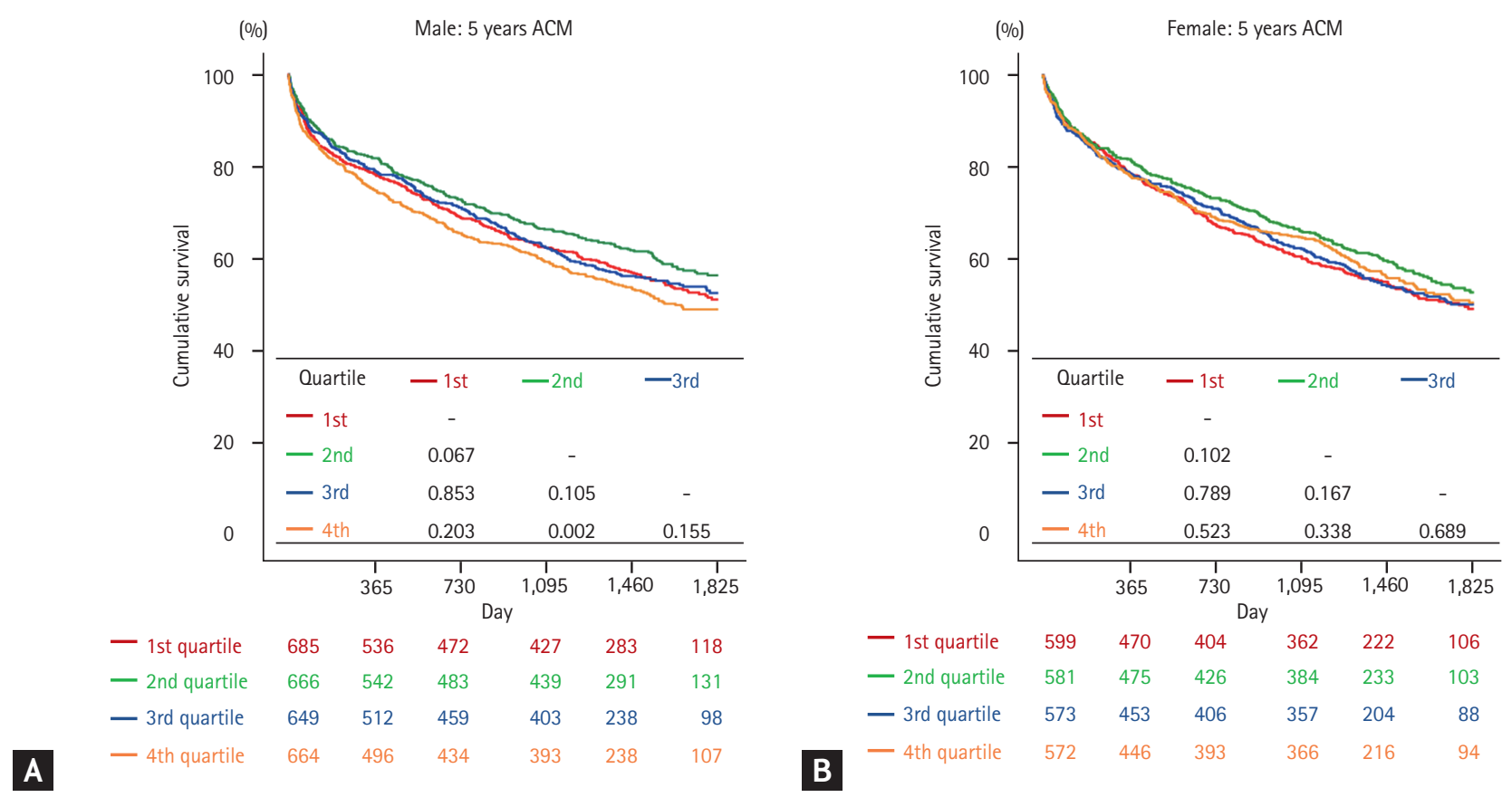

Figure 2. Five-year outcomes according to corrected QT quartiles in acute heart failure patients. The Kaplan-Meier curves showed 5-year all-cause survival rates in (A) male and (B) female. ACM, all-cause mortality.

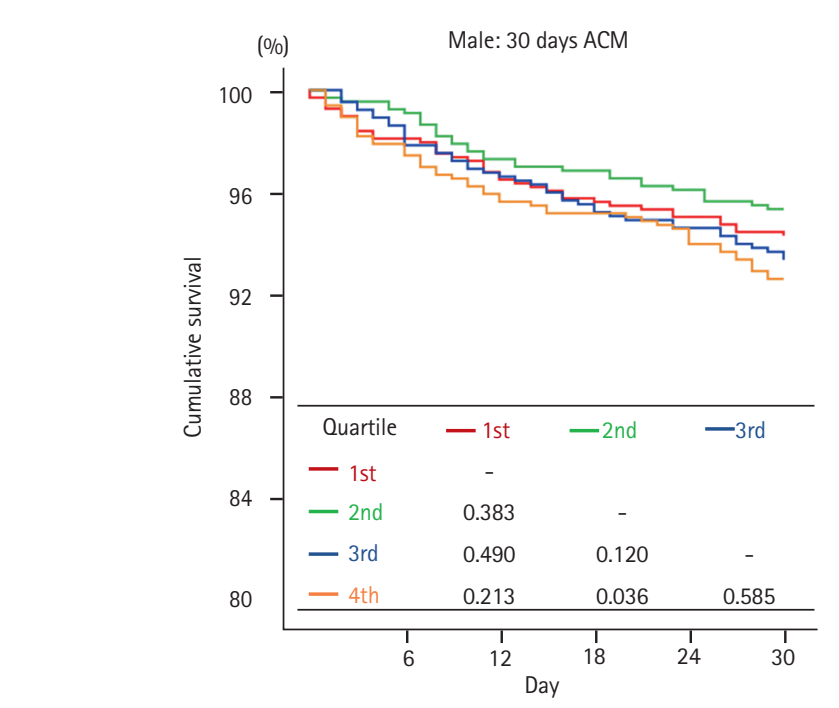

$\begin{array}{llllllll} & \text { 1st quartile } & 685 & 671 & 661 & 654 & 649 & 645 \\ & \text { 2nd quartile } & 666 & 661 & 647 & 643 & 637 & 632 \\ \text { 3rd quartile } & 649 & 639 & 626 & 616 & 610 & 604 \\ \text { A } & \text { 4th quartile } & 664 & 649 & 634 & 629 & 615 & 612\end{array}$

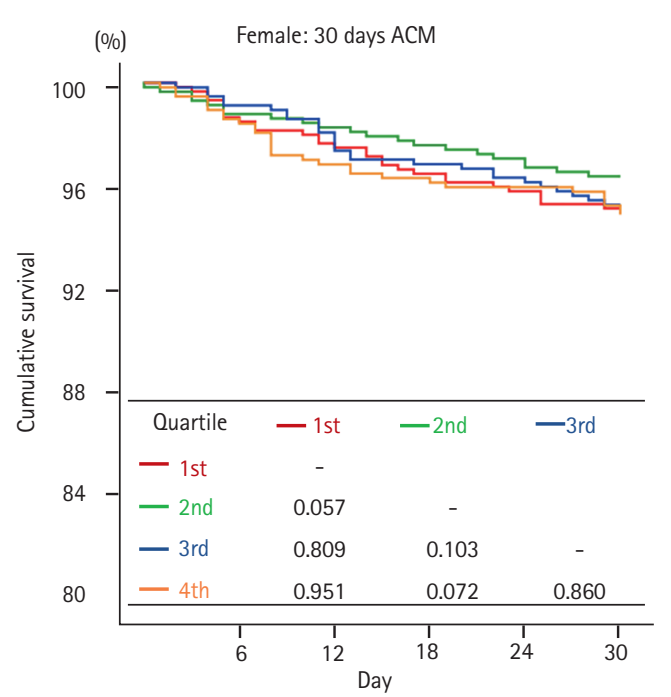

$\begin{array}{lllllll}\text { 1st quartile } & 599 & 590 & 583 & 576 & 572 & 568 \\ \text { 2nd quartile } & 581 & 574 & 571 & 567 & 564 & 560 \\ \text { 3rd quartile } & 573 & 568 & 562 & 555 & 552 & 546\end{array}$

B $\quad$ 4th quartile $\quad 572 \quad 563 \quad 551 \quad 548 \quad 545 \quad 541$

Figure 3. Thirty-day outcomes according to corrected QT quartiles in acute heart failure patients. The Kaplan-Meier curves showed 30-day all-cause survival rates in (A) male and (B) female. ACM, all-cause mortality. 
Table 3. Unadjusted and adjusted Cox regression analysis for 5-year all-cause death

\begin{tabular}{|c|c|c|c|c|c|c|}
\hline \multirow{2}{*}{ Variable } & \multicolumn{2}{|c|}{ Univariate analysis } & \multicolumn{2}{|l|}{ Model $1^{a}$} & \multicolumn{2}{|l|}{ Model $2^{b}$} \\
\hline & $\mathrm{HR}(95 \% \mathrm{CI})$ & $p$ & $\mathrm{HR}(95 \% \mathrm{CI})$ & $p$ & $\mathrm{HR}(95 \% \mathrm{CI})$ & $p$ \\
\hline \multicolumn{7}{|l|}{ Male } \\
\hline ıst quartile & $1.166(0.991-1.373)$ & 0.065 & $1.164(0.988-1.371)$ & 0.069 & $1.158(0.981-1.366)$ & 0.084 \\
\hline 2nd quartile & Reference & & Reference & & Reference & \\
\hline 3rd quartile & $1.148(0.972-1.355)$ & 0.105 & $1.142(0.967-1.349)$ & 0.118 & $1.185(1.001-1.404)$ & 0.049 \\
\hline 4th quartile & $1.289(1.095-1.516)$ & 0.002 & $1.252(1.058-1.481)$ & 0.009 & $1.404(1.091-1.535)$ & 0.003 \\
\hline \multicolumn{7}{|l|}{ Female } \\
\hline 1st quartile & $1.152(0.972-1.366)$ & 0.102 & $1.148(0.968-1.361)$ & 0.113 & $1.089(0.916-1.295)$ & 0.332 \\
\hline 2nd quartile & Reference & & Reference & & Reference & \\
\hline 3rd quartile & $1.128(0.949-1.340)$ & 0.171 & $1.136(0.956-1.351)$ & 0.147 & $1.072(0.901-1.276)$ & 0.433 \\
\hline 4th quartile & $1.089(0.915-1.296)$ & 0.335 & $1.074(0.895-1.290)$ & 0.442 & $1.021(0.849-1.229)$ & 0.824 \\
\hline
\end{tabular}

HR, hazard ratio; CI, confidence interval.

${ }^{a}$ Multivariate analysis were calculated by Cox regression models, including atrial fibrillation and right or left bundle branch block.

${ }^{\mathrm{b}}$ Multivariate analysis were calculated by Cox regression models, including age, body mass index, previous history of hypertension, diabetes mellitus, heart failure, ischemic heart disease, valvular heart disease, chronic obstructive lung disease, chronic renal failure, cerebrovascular accident, atrial fibrillation, beta-blocker medication at discharge, and right or left bundle branch block.

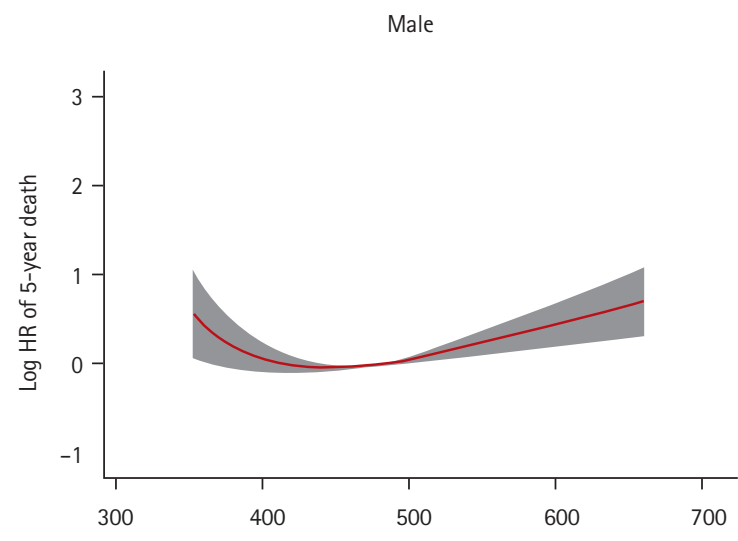

A

QTc interval

Figure 4. Thirty-day outcomes according to corrected QT (QTc) quartiles in acute heart failure patients. The Kaplan-Meier curves showed 30 -day all-cause survival rates in (A) male and (B) female. HR, hazard ratio.

\section{DISCUSSION}

The major findings of this study are as follows: (1) A substantial number of patients with acute HF had prolonged QTc interval (QTc $\geq 440 \mathrm{~ms}$ ) than previously considered normal range; (2) in Kaplan-Meier analysis, prolonged QTc interval increases the 5-year mortality and 30-day mortality in male but not in female; (3) after adjusting covariates, QTc prolongation had independent prognostic impact only in male, but its significance was attenuated in female; (4) there was J-shape association between 5-year mortality in QTc interval in both male and female.

These findings were in congruence with previous 
Table 4. Unadjusted and adjusted Cox regression analysis for 30-day all-cause death

\begin{tabular}{|c|c|c|c|c|c|c|}
\hline \multirow{2}{*}{ Variable } & \multicolumn{2}{|c|}{ Univariate analysis } & \multicolumn{2}{|l|}{ Model $1^{a}$} & \multicolumn{2}{|l|}{ Model $2^{b}$} \\
\hline & $\mathrm{HR}(95 \% \mathrm{CI})$ & $p$ value & $\operatorname{HR}(95 \% \mathrm{CI})$ & $p$ value & $\mathrm{HR}(95 \% \mathrm{CI})$ & $p$ value \\
\hline \multicolumn{7}{|l|}{ Male } \\
\hline 1st quartile & $1.232(0.769-1.974)$ & 0.386 & $1.280(0.798-2.052)$ & 0.306 & $1.174(0.730-1.889)$ & 0.507 \\
\hline 2nd quartile & Reference & & Reference & & Reference & \\
\hline 3rd quartile & $1.437(0.906-2.281)$ & 0.124 & $1.404(0.884-2.230)$ & 0.151 & $1.336(0.837-2.132)$ & 0.225 \\
\hline 4th quartile & $1.610(1.027-2.525)$ & 0.038 & $1.432(0.896-2.290)$ & 0.133 & $1.368(0.851-2.199)$ & 0.196 \\
\hline \multicolumn{7}{|l|}{ Female } \\
\hline 1st quartile & $1.350(0.770-2.368)$ & 0.295 & $1.368(0.780-2.400)$ & 0.274 & $1.131(0.639-2.001)$ & 0.674 \\
\hline 2nd quartile & Reference & & Reference & & Reference & \\
\hline 3rd quartile & $1.307(0.739-2.311)$ & $0.35^{8}$ & $1.280(0.723-2.266)$ & 0.398 & $1.221(0.689-2.164)$ & 0.493 \\
\hline 4th quartile & $1.420(0.810-2.490)$ & 0.221 & $1.355(0.753-2.440)$ & 0.311 & $1.338(0.744-2.408)$ & 0.331 \\
\hline
\end{tabular}

HR, hazard ratio; CI, confidence interval.

${ }^{\mathrm{a}}$ Multivariate analysis were calculated by Cox regression models, including atrial fibrillation and right or left bundle branch block.

${ }^{\mathrm{b}}$ Multivariate analysis were calculated by Cox regression models, including age, body mass index, previous history of hypertension, diabetes mellitus, heart failure, ischemic heart disease, valvular heart disease, chronic obstructive lung disease, chronic renal failure, cerebrovascular accident, atrial fibrillation, beta-blocker medication at discharge, and right or left bundle branch block.

studies which demonstrated increased mortality risk in chronic HF patients with prolonged QT interval [22,23]. Furthermore, our study found that a substantial number of patients had prolonged QTc interval, and the nadir of J-shaped association was significantly right-shifted in patients with acute HF compared to those with chronic HF [22]. This discrepancy could have influenced the results of a previous study that reported lack of prognostic impact of QTc interval and absence of of J-shaped association, as aforementioned study analyzed the QTc duration only by a binary classification based on the 440 ms threshold [24]. We did not explore the underlying biology linking QTc interval and mortality in this study. To our knowledge, there is a paucity of data which could directly explain this J-shaped relationship. Based on the findings of several experimental studies, the authors cautiously suggest that a relatively short QTc duration and repolarization time could be a substrate for multiple-circuit reentry excitation, which would induce atrial/ventricular arrhythmia [25,26].

Right shifted QTc interval range with low risk of allcause mortality, as shown in the lowest mortality of and quartile, might be attributed to the complex characteristics of acute HF. Patients with acute HF experience a decline in pumping capacity, which results in hypoperfusion and congestion. Compensatory pathways, including the renin-angiotensin system and sympathetic nervous system, are then activated to maintain cardiac output by increasing salt retention and augmented cardiac contractility $[27,28]$. Although sustained compensatory mechanisms could lead to pathologic end-organ damage, their activation is important for the restoration of the cardiovascular status within the hemodynamically stable physiologic range and renders patients asymptomatic in the short acute period [24]. The QTc interval represents the time of electrical depolarization and repolarization of ventricular myocardium. However, as shown in Table 3, QTc interval persistently demonstrated prognostic implications even after adjusting for multiple comorbidities, including left ventricular end diastolic diameter. Therefore, we hypothesize that QTc interval could be influenced by various environmental factors, in addition to the left ventricular dimension. Studies have demonstrated that the QTc interval could vary according to sympathetic and parasympathetic tone [29,30]. In particular, increased catecholamine levels, which could often be observed in patients with acute heart failure, have been reported to induce QTc interval 
prolongation [31-33]. Change in QTc interval in patients with acute HF might be confounded by its complex pathophysiologic status, not only with respect to structural abnormality, but also with respect to adaptively activated neurohormonal pathways, which were different from those in participants of other studies.

A variety of studies have reported significantly longer QTc intervals in females compared to their male counterparts [34,35], in those of normal status as well as those with various pathologies $[36,37]$. These studies suggested that sex hormone differences between male and female could have an influence on ventricular repolarization. Therefore, we postulated that the QTc interval of females might be longer than that of males among patients with AHF, and set the quartiles separately for males and females.

We found that prolonged QTc interval has a prognostic implication in males, but its significance was diminished in females. Although we did not fully explore the underlying biological mechanisms, there are several possible explanations for our results. Female patients showed a higher percentage of heart failure with preserved ejection fraction (HFpEF) than male patients. When we analyzed the multifractional polynomial Cox regression according to HF phenotypes, we found that the J-curve association was steeper in the upper limits in patients with heart failure with reduced ejection fraction compared to those with HFpEF. In addition, female patients were older and showed a preponderance of a previous history of hypertension, heart failure and valvular heart disease (Supplementary Table 1), which were frequently observed in deceased subjects (Supplementary Table 2). Considering these together, we cautiously hypothesize that these detrimental covariates might attenuate the statistical significance of the QTc interval in female patients.

In this study, we adopted the QTc interval measured and calculated automatically by standard commercial electrographs. However, several previous studies on QT or QTc interval measured the duration of QT interval manually $[12,22,24,38,39]$. These manual methods are not real-world clinical practices, are time-consuming processes, and demonstrated physician dependency (even among trained cardiologists) [40,41]. In addition, automatic method has recently proved its usefulness and accuracy in QT interval measurement [42,43].
This study provides an insight into the association between QTc duration and all-cause mortality. While accurate cause of the right-shifted relation cannot be fully understood through our report, our findings have valuable clinical implications. QTc duration can be easily obtained and measured with a 12-lead ECG or even mobile devices. However, such simple measurement can predict the prognosis in high risk patients. It could be helpful to stratify the risk of patients in addition to other risk factors without invasive procedure [8,9]; for example, it might be applied in tele-monitoring.

Some limitations of this study must be taken into consideration. First, the onset of prolonged QTc and the effect of dynamic QTc interval change during follow-up were unknown in our registry. Second, we did not analyze the confounding effect of antiarrhythmic agents such as amiodarone for atrial fibrillation and antidepressants, which might induce QTc interval prolongation [21,22]. However, the J-shaped relationship of QTc interval and all-cause mortality persisted regardless of atrial fibrillation, which accounted for most type III medication use. This issue appeared minor. In addition, since the patients in this study all come from East Asia, it is unknown whether the results of this study can be extrapolated to other ethnicities and countries. Fourth, we used QTc data obtained by real-world 12-lead ECG devices. This study was intended to show the practical prognostic value of the QTc interval, but there could be confounders in patients with atrial fibrillation and bundle branch block, although these factors were adjusted for in the multivariable analysis. In addition, since we did not assess all echocardiographic parameters regarding LV mass, we could not investigate the association between QTc interval and LV mass index. Lastly, we excluded patients with pacing rhythm, as their activation and deactivation pathway might be different from that of those without artificial pacing.

In conclusion, a substantial number of patients with AHF had prolonged QTc interval. QTc interval is an independent prognosticator for 5-year mortality in male, but it failed to show prognostic significance in female. The relationship between QTc interval and all-cause mortality was J-shaped as was previously known; however, it seemed to be right-shifted in patients with AHF and less steeper in female. 


\section{KEY MESSAGE}

1. More than three-fourths of patients with acute heart failure had prolonged corrected QT (QTc) interval (QTc $\geq 440 \mathrm{~ms}$ ) than previously considered normal range.

2. In Kaplan-Meier analysis, 4th QTc quartile demonstrated increased 5-year mortality and 30-day mortality in male but not in female.

3. After adjusting covariates, QTc prolongation had independent prognostic impact only in male, but its significance was attenuated in female.

4. There was J-shape association between 5-year mortality in QTc interval in both male (nadir 440 to $450 \mathrm{~ms}$ ) and female ( 470 to $480 \mathrm{~ms}$ ).

\section{Conflict of interest}

No potential conflict of interest relevant to this article was reported.

\section{Acknowledgments}

This work was supported by Research of Korea Centers for Disease Control and Prevention (2010-E63003-00, 2011-E63002-00, 2012-E63005-00, and 2013-E63003-00).

The KorAHF Registry was conducted from 10 tertiary medical centers: Seoul National University Hospital, Seoul, Korea; Sungkyunkwan University College of Medicine, Seoul, Korea; University of Ulsan College of Medicine, Seoul, Korea; Chungbuk National University College of Medicine, Cheongju, Korea; Kyungpook National University College of Medicine, Daegu, Korea; The Catholic University of Korea, Seoul, Korea; Yonsei University College of Medicine, Seoul, Korea; Yonsei University Wonju College of Medicine, Wonju, Korea; Seoul National University Bundang Hospital, Seongnam, Korea; Heart Research Center of Chonnam National University, Gwangju, Korea.

\section{REFERENCES}

1. Benjamin EJ, Virani SS, Callaway CW, et al. Heart disease and stroke statistics-2018 update: a report from the American Heart Association. Circulation 2018;137:e67-e492.
2. Lee SE, Cho HJ, Lee HY, et al. A multicentre cohort study of acute heart failure syndromes in Korea: rationale, design, and interim observations of the Korean Acute Heart Failure (KorAHF) registry. Eur J Heart Fail 2014;16:700708.

3. Lee SE, Lee HY, Cho HJ, et al. Clinical characteristics and outcome of acute heart failure in Korea: results from the Korean Acute Heart Failure Registry (KorAHF). Korean Circ J 2017;47:341-353.

4. Youn JC, Han S, Ryu KH. Temporal trends of hospitalized patients with heart failure in Korea. Korean Circ J 2017;47:16-24.

5. Nieminen MS, Brutsaert D, Dickstein K, et al. EuroHeart Failure Survey II (EHFS II): a survey on hospitalized acute heart failure patients: description of population. Eur Heart J 2006;27:2725-2736.

6. Jong P, Vowinckel E, Liu PP, Gong Y, Tu JV. Prognosis and determinants of survival in patients newly hospitalized for heart failure: a population-based study. Arch Intern Med 2002;162:1689-1694.

7. Stewart S, MacIntyre K, Hole DJ, Capewell S, McMurray JJ. More 'malignant' than cancer? Five-year survival following a first admission for heart failure. Eur J Heart Fail 2001;3:315-322.

8. Novack V, Pencina M, Zahger D, et al. Routine laboratory results and thirty day and one-year mortality risk following hospitalization with acute decompensated heart failure. PLoS One 2010;5:e12184.

9. Rudiger A, Burckhardt OA, Harpes P, Muller SA, Follath F. The relative lymphocyte count on hospital admission is a risk factor for long-term mortality in patients with acute heart failure. Am J Emerg Med 2006;24:451-454.

10. Yancy CW, Jessup M, Bozkurt B, et al. 2013 ACCF/AHA guideline for the management of heart failure: a report of the American College of Cardiology Foundation/American Heart Association Task Force on Practice Guidelines. J Am Coll Cardiol 2013;62:e147-e239.

11. Ponikowski P, Voors AA, Anker SD, et al. 2016 ESC guidelines for the diagnosis and treatment of acute and chronic heart failure: the task force for the diagnosis and treatment of acute and chronic heart failure of the European Society of Cardiology (ESC) Developed with the special contribution of the Heart Failure Association (HFA) of the ESC. Eur Heart J 2016;37:2129-2200.

12. Goldenberg I, Moss AJ, Zareba W. QT interval: how to measure it and what is "normal". J Cardiovasc Electro- 
physiol 2006;17:333-336.

13. Gaudron P, Kugler I, Hu K, Bauer W, Eilles C, Ertl G. Time course of cardiac structural, functional and electrical changes in asymptomatic patients after myocardial infarction: their inter-relation and prognostic impact. J Am Coll Cardiol 2001;38:33-40.

14. Heemskerk CPM, Pereboom M, van Stralen K, et al. Risk factors for QTc interval prolongation. Eur J Clin Pharmacol 2018;74:183-191.

15. Elming H, Holm E, Jun L, et al. The prognostic value of the QT interval and QT interval dispersion in all-cause and cardiac mortality and morbidity in a population of Danish citizens. Eur Heart J 1998;19:1391-1400.

16. Day CP, McComb JM, Campbell RW. QT dispersion: an indication of arrhythmia risk in patients with long QT intervals. Br Heart J 1990;63:342-344.

17. Okin PM, Devereux RB, Howard BV, Fabsitz RR, Lee ET, Welty TK. Assessment of QT interval and QT dispersion for prediction of all-cause and cardiovascular mortality in American Indians: The Strong Heart Study. Circulation 2000;101:61-66.

18. Schouten EG, Dekker JM, Meppelink P, Kok FJ, Vandenbroucke JP, Pool J. QT interval prolongation predicts cardiovascular mortality in an apparently healthy population. Circulation 1991;84:1516-1523.

19. Pickham D, Helfenbein E, Shinn JA, et al. High prevalence of corrected QT interval prolongation in acutely ill patients is associated with mortality: results of the QT in Practice (QTIP) Study. Crit Care Med 2012;40:394-399.

20. Straus SM, Kors JA, De Bruin ML, et al. Prolonged QTc interval and risk of sudden cardiac death in a population of older adults. J Am Coll Cardiol 2006;47:362-367.

21. Zhang Y, Post WS, Dalal D, Blasco-Colmenares E, Tomaselli GF, Guallar E. QT-interval duration and mortality rate: results from the Third National Health and Nutrition Examination Survey. Arch Intern Med 2011;171:17271733 .

22. Brooksby $\mathrm{P}$, Batin PD, Nolan J, et al. The relationship between QT intervals and mortality in ambulant patients with chronic heart failure. The United Kingdom heart failure evaluation and assessment of risk trial (UKHEART). Eur Heart J 1999;20:1335-1341.

23. Vrtovec B, Delgado R, Zewail A, Thomas CD, Richartz BM, Radovancevic B. Prolonged QTc interval and high B-type natriuretic peptide levels together predict mortality in patients with advanced heart failure. Circulation
2003;107:1764-1769.

24. Breidthardt T, Christ M, Matti M, et al. QRS and QTc interval prolongation in the prediction of long-term mortality of patients with acute destabilised heart failure. Heart 2007;93:1093-1097.

25. Greener ID, Monfredi O, Inada S, et al. Molecular architecture of the human specialised atrioventricular conduction axis. J Mol Cell Cardiol 2011;50:642-651.

26. Nattel S. New ideas about atrial fibrillation 50 years on. Nature 2002;415:219-226.

27. Floras JS. Sympathetic nervous system activation in human heart failure: clinical implications of an updated model. J Am Coll Cardiol 2009;54:375-385.

28. Lindgren L, Yli-Hankala A, Randell T, Kirvela M, Scheinin M, Neuvonen PJ. Haemodynamic and catecholamine responses to induction of anaesthesia and tracheal intubation: comparison between propofol and thiopentone. $\mathrm{Br} \mathrm{J}$ Anaesth 1993;70:306-310.

29. Nielsen JB, Graff C, Pietersen A, et al. J-shaped association between QTc interval duration and the risk of atrial fibrillation: results from the Copenhagen ECG study. J Am Coll Cardiol 2013;61:2557-2564.

30. Beinart R, Zhang Y, Lima JA, et al. The QT interval is associated with incident cardiovascular events: the MESA study. J Am Coll Cardiol 2014;64:2111-2119.

31. Baumert M, Lambert GW, Dawood T, et al. QT interval variability and cardiac norepinephrine spillover in patients with depression and panic disorder. Am J Physiol Heart Circ Physiol 2008;295:H962-H968.

32. Kaufman ES, Gorodeski EZ, Dettmer MM, Dikshteyn M. Use of autonomic maneuvers to probe phenotype/genotype discordance in congenital long QT syndrome. Am J Cardiol 2005;96:1425-1430.

33. Vyas H, Hejlik J, Ackerman MJ. Epinephrine QT stress testing in the evaluation of congenital long-QT syndrome: diagnostic accuracy of the paradoxical QT response. Circulation 2006;113:1385-1392.

34. Rautaharju PM, Zhou SH, Wong S, et al. Sex differences in the evolution of the electrocardiographic QT interval with age. Can J Cardiol 1992;8:690-695.

35. Pham TV, Rosen MR. Sex, hormones, and repolarization. Cardiovasc Res 2002;53:740-751.

36. Chauhan VS, Krahn AD, Walker BD, Klein GJ, Skanes AC, Yee R. Sex differences in QTc interval and QT dispersion: dynamics during exercise and recovery in healthy subjects. Am Heart J 2002;144:858-864. 
37. Shin HS, Lee WY, Kim SW, et al. Sex difference in the relationship between insulin resistance and corrected QT interval in non-diabetic subjects. Circ J 2005;69:409-413.

38. Lecaillon JB, Kaiser G, Palmisano M, Morgan J, Della Cioppa G. Pharmacokinetics and tolerability of formoterol in healthy volunteers after a single high dose of Foradil dry powder inhalation via Aerolizer. Eur J Clin Pharmacol 1999:55:131-138.

39. Velavan P, Khan NK, Goode K, et al. Predictors of short term mortality in heart failure: insights from the Euro Heart Failure survey. Int J Cardiol 2010;138:63-69.

40. Mangoni AA, Kinirons MT, Swift CG, Jackson SH. Impact of age on QT interval and QT dispersion in healthy subjects: a regression analysis. Age Ageing 2003;32:326-331.
41. Anderson ME, Al-Khatib SM, Roden DM, Califf RM; Duke Clinical Research Institute/American Heart Journal Expert Meeting on Repolarization Changes. Cardiac repolarization: current knowledge, critical gaps, and new approaches to drug development and patient management. Am Heart J 2002;144:769-781.

42. Viskin S, Rosovski U, Sands AJ, et al. Inaccurate electrocardiographic interpretation of long QT: the majority of physicians cannot recognize a long QT when they see one. Heart Rhythm 2005;2:569-574.

43. Malik M, Farbom P, Batchvarov V, Hnatkova K, Camm AJ. Relation between QT and RR intervals is highly individual among healthy subjects: implications for heart rate correction of the QT interval. Heart 2002;87:220-228. 
Supplementary Table 1. Baseline characteristics according to $\operatorname{sex}($ total $n=4,990)$

\begin{tabular}{|c|c|c|c|}
\hline Characteristic & Male $(n=2,665)$ & Female $(n=2,325)$ & $p$ value \\
\hline \multicolumn{4}{|l|}{ Demographic data } \\
\hline Age, yr & $65.3 \pm 14.8$ & $71.8 \pm 13.8$ & $<0.001$ \\
\hline Body mass index, $\mathrm{kg} / \mathrm{m}^{2}$ & $23.6 \pm 3.8$ & $23.1 \pm 4.0$ & $<0.001$ \\
\hline \multicolumn{4}{|l|}{ Past medical history } \\
\hline Hypertension & $1,486(55.8)$ & $1,483(63.8)$ & $<0.001$ \\
\hline Diabetes mellitus & $996(36.2)$ & $83(35 \cdot 7)$ & 0.710 \\
\hline Heart failure & $1,093(41.0)$ & $1,021(43 \cdot 9)$ & 0.039 \\
\hline Ischemic heart disease & $853(32.0)$ & $582(25.0)$ & $<0.001$ \\
\hline Valvular heart disease & $309(11.6)$ & $420(18.1)$ & $<0.001$ \\
\hline Chronic obstructive lung disease & $352(13.2)$ & $197(8.5)$ & $<0.001$ \\
\hline Chronic kidney disease & $414(15 \cdot 5)$ & $284(12.2)$ & 0.001 \\
\hline Cerebrovascular accident & $429(16.1)$ & $342(14 \cdot 7)$ & 0.174 \\
\hline \multicolumn{4}{|l|}{ Social history } \\
\hline Smoking history & & & $<0.001$ \\
\hline Current smoker & $826(31.0)$ & $110(4.7)$ & \\
\hline Ex-smoker & $942(35 \cdot 3)$ & $76(3 \cdot 3)$ & \\
\hline Never smoker & $897(33.7)$ & $2,139(92.0)$ & \\
\hline \multicolumn{4}{|l|}{ Alcohol history } \\
\hline Heavy drinker & $327(12.3)$ & $22(0.9)$ & \\
\hline Social drinker & $1,289(48.4)$ & $291(12.5)$ & \\
\hline Never drinker & $1,049(39.4)$ & $2,012(86.5)$ & \\
\hline \multicolumn{4}{|l|}{ Major ECG abnormalities } \\
\hline Atrial fibrillation & $703(26.4)$ & $689(29.6)$ & 0.011 \\
\hline Right bundle branch block & $222(8.3)$ & $138(5 \cdot 9)$ & 0.001 \\
\hline Left bundle branch block & $119(4 \cdot 5)$ & $150(6.5)$ & 0.002 \\
\hline NYHA functional class & & & 0.013 \\
\hline Stage II & $437(16.4)$ & $321(13.8)$ & \\
\hline Stage III & $978(36.7)$ & $835(35 \cdot 9)$ & \\
\hline Stage IV & $1,250(46.9)$ & $1,169(50.3)$ & \\
\hline \multicolumn{4}{|l|}{ Physical exam } \\
\hline $\mathrm{SBP}, \mathrm{mmHg}$ & $130.1 \pm 30.7$ & $134 \cdot 3 \pm 29 \cdot 9$ & $<0.001$ \\
\hline $\mathrm{DBP}, \mathrm{mmHg}$ & $78.9 \pm 19.3$ & $79.3 \pm 18.4$ & 0.403 \\
\hline HR, beats/min & $92.2 \pm 25.8$ & $93 \cdot 4 \pm 25 \cdot 6$ & 0.110 \\
\hline \multicolumn{4}{|l|}{ Laboratory exam } \\
\hline Hemoglobin, mg/dL & $14.6 \pm 2.2$ & $14.7 \pm 1.9$ & 0.272 \\
\hline Sodium, mmol/L & $137.6 \pm 4.6$ & $137.6 \pm 5.0$ & 0.681 \\
\hline Potassium, mmol/L & $4.4 \pm 0.7$ & $4.3 \pm 0.7$ & $<0.001$ \\
\hline BUN, mg/dL & $26.5 \pm 16.4$ & $25.4 \pm 16.2$ & 0.013 \\
\hline Creatinine, mg/dL & $1.6 \pm 1.7$ & $1.3 \pm 1.2$ & $<0.001$ \\
\hline BNP, pg/mL & $854.0(443.0-1,667.0)$ & $936.0(504.4-1,821.8)$ & 0.115 \\
\hline NT-proBNP, pg/mL & $4,349 \cdot 0(1,975 \cdot 0-10,301.0)$ & $5,366.0(2,379.0-13,763.0)$ & $<0.001$ \\
\hline
\end{tabular}


Supplementary Table 1. Continued

\begin{tabular}{|c|c|c|c|}
\hline Characteristic & Male $(n=2,665)$ & Female $(\mathrm{n}=2,325)$ & $p$ value \\
\hline Beta-blockers & $716(26.9)$ & $704(30.3)$ & 0.008 \\
\hline Renin-angiotensin-system inhibitors & $1,010(37.9)$ & $880(37.8)$ & 0.971 \\
\hline Mineralocorticoid receptor blockers & $459(17.2)$ & $451(19.4)$ & 0.047 \\
\hline \multicolumn{4}{|l|}{ Medication at discharge } \\
\hline Beta-blockers & $1,330(49.9)$ & $1,090(46.9)$ & 0.033 \\
\hline Renin-angiotensin-system inhibitors & $1,820(68.3)$ & $1,510(64 \cdot 9)$ & 0.012 \\
\hline Mineralocorticoid receptor blockers & $1,187(44 \cdot 5)$ & $1,066(45.8)$ & 0.354 \\
\hline \multicolumn{4}{|l|}{ Echocardiographic findings } \\
\hline Left ventricular end diastolic diameter, mm & $60.1 \pm 9.9$ & $54 \cdot 4 \pm 9 \cdot 3$ & $<0.001$ \\
\hline Ejection fraction, \% & $34.6 \pm 14.6$ & $41.4 \pm 15.8$ & $<0.001$ \\
\hline $\begin{array}{l}\text { Heart failure with reduced ejection fraction } \\
(<50 \%)\end{array}$ & $2,150(83.7)$ & $1,531(68.3)$ & $<0.001$ \\
\hline
\end{tabular}

Values are presented as mean $\pm \mathrm{SD}$, number (\%), or median (interquartile range).

ECG, electrocardiogram; NYHA, New York Heart association; SBP, systolic blood pressure; DBP, diastolic blood pressure; HR, heart rate; BUN, blood urea nitrogen; BNP, B-type natriuretic peptide; NT-proBNP, N-terminal proBNP. 
Supplementary Table 2. Baseline characteristics of subjects according to 5 -year survival (total $n=4,990)$

\begin{tabular}{|c|c|c|c|}
\hline Characteristic & Alive $(\mathrm{n}=2,747)$ & Deceased $(n=2,243)$ & $p$ value \\
\hline \multicolumn{4}{|l|}{ Demographic data } \\
\hline Age, yr & $63.7 \pm 15.0$ & $74.0 \pm 12.2$ & $<0.001$ \\
\hline Sex, men & $1,480(53.9)$ & $1,185(52.8)$ & 0.461 \\
\hline Body mass index, $\mathrm{kg} / \mathrm{m}^{2}$ & $24.1 \pm 3.9$ & $22.5 \pm 3.7$ & $<0.001$ \\
\hline \multicolumn{4}{|l|}{ Past medical history } \\
\hline Hypertension & $1,474(53.7)$ & $1,495(66.7)$ & $<0.001$ \\
\hline Diabetes mellitus & $864(31.5)$ & $933(41.6)$ & $<0.001$ \\
\hline Heart failure & $930(33.9)$ & $1,184(52.8)$ & $<0.001$ \\
\hline Ischemic heart disease & $615(22.4)$ & $820(36.6)$ & $<0.001$ \\
\hline Valvular heart disease & $376(13.7)$ & $353(15.7)$ & 0.042 \\
\hline Chronic obstructive lung disease & $242(8.8)$ & $307(13.7)$ & $<0.001$ \\
\hline Chronic kidney disease & $232(8.4)$ & $466(20.8)$ & $<0.001$ \\
\hline Cerebrovascular accident & $310(11.3)$ & $461(20.6)$ & $<0.001$ \\
\hline \multicolumn{4}{|l|}{ Social history } \\
\hline Smoking history & & & $<0.001$ \\
\hline Current smoker & $552(37 \cdot 3)$ & $274(23.1)$ & \\
\hline Ex-smoker & $472(31.9)$ & $470(39.7)$ & \\
\hline Never smoker & $456(30.8)$ & $441(37.2)$ & \\
\hline Alcohol history & & & $<0.001$ \\
\hline Heavy drinker & $233(15.1)$ & $104(8.8)$ & \\
\hline Social drinker & $780(52.7)$ & $509(43.0)$ & \\
\hline Never drinker & $477(32.2)$ & $572(48.3)$ & \\
\hline \multicolumn{4}{|l|}{ Major ECG abnormalities } \\
\hline Atrial fibrillation & $965(35.1)$ & $804(35.8)$ & 0.599 \\
\hline Right bundle branch block & $188(6.8)$ & $172(7.7)$ & 0.263 \\
\hline Left bundle branch block & $138(5.0)$ & $131(5.8)$ & 0.204 \\
\hline NYHA functional class & & & $<0.001$ \\
\hline Stage II & $515(18.7)$ & $243(10.8)$ & \\
\hline Stage III & $1,039(37.8)$ & $774(34.5)$ & \\
\hline Stage IV & $1,193(43 \cdot 4)$ & $1,226(54 \cdot 7)$ & \\
\hline \multicolumn{4}{|l|}{ Physical exam } \\
\hline $\mathrm{SBP}, \mathrm{mmHg}$ & $132.5 \pm 29.9$ & $131.5 \pm 31.0$ & 0.255 \\
\hline DBP, mmHg & $80.5 \pm 19.1$ & $77 \cdot 3 \pm 18.4$ & $<0.001$ \\
\hline HR, beats per min & $93.5 \pm 26.2$ & $91.9 \pm 25.1$ & 0.037 \\
\hline \multicolumn{4}{|l|}{ Laboratory exam } \\
\hline Hemoglobin, mg/dL & $14.3 \pm 1.9$ & $15.1 \pm 2.1$ & $<0.001$ \\
\hline Sodium, mmol/L & $138.3 \pm 4.3$ & $136.7 \pm 5.2$ & $<0.001$ \\
\hline Potassium, mmol/L & $4.3 \pm 0.6$ & $4.5 \pm 0.8$ & $<0.001$ \\
\hline $\mathrm{BUN}, \mathrm{mg} / \mathrm{dL}$ & $22.6 \pm 13.8$ & $30.1 \pm 18.2$ & $<0.001$ \\
\hline Creatinine, mg/dL & $1.3 \pm 1.4$ & $1.7 \pm 1.5$ & $<0.001$ \\
\hline BNP, pg/mL & $728.1(392.7-1,341.8)$ & $1,117.3(595.6-2,289.0)$ & $<0.001$ \\
\hline NT-proBNP, pg/mL & $3,541.0(1,561.0-7,627.8)$ & $7,222.0(3,342.3-17,506.2)$ & $<0.001$ \\
\hline
\end{tabular}


Supplementary Table 2. Continued

\begin{tabular}{|c|c|c|c|}
\hline Characteristic & Alive $(\mathrm{n}=2,747)$ & Deceased $(n=2,243)$ & $p$ value \\
\hline \multicolumn{4}{|l|}{ Medication at admission } \\
\hline Beta-blockers & $734(26.7)$ & $686(30.6)$ & 0.003 \\
\hline Renin-angiotensin-system inhibitors & $966(35.2)$ & $924(41.2)$ & $<0.001$ \\
\hline Mineralocorticoid receptor blockers & $435(15.8)$ & $475(21.2)$ & $<0.001$ \\
\hline \multicolumn{4}{|l|}{ Medication at discharge } \\
\hline Beta-blockers & $1,493(54 \cdot 4)$ & $927(41.3)$ & $<0.001$ \\
\hline Renin-angiotensin-system inhibitors & $1,964(71.5)$ & $1,366(60.9)$ & $<0.001$ \\
\hline Mineralocorticoid receptor blockers & $1,315(47 \cdot 9)$ & $938(41.8)$ & $<0.001$ \\
\hline \multicolumn{4}{|l|}{ Echocardiographic findings } \\
\hline Left ventricular end diastolic diameter, $\mathrm{mm}$ & $57.8 \pm 9.9$ & $57.0 \pm 10.3$ & 0.005 \\
\hline Ejection fraction, \% & $37.8 \pm 15 \cdot 7$ & $37 \cdot 7 \pm 15 \cdot 3$ & 0.888 \\
\hline $\begin{array}{l}\text { Heart failure with reduced ejection fraction } \\
(<50 \%)\end{array}$ & $2,035(75 \cdot 9)$ & $1,646(77 \cdot 3)$ & 0.265 \\
\hline
\end{tabular}

Values are presented as mean $\pm \mathrm{SD}$, number (\%), or median (interquartile range).

ECG, electrocardiogram; NYHA, New York Heart association; SBP, systolic blood pressure; DBP, diastolic blood pressure; HR, heart rate; BUN, blood urea nitrogen; BNP, B-type natriuretic peptide; NT-proBNP, N-terminal proBNP. 

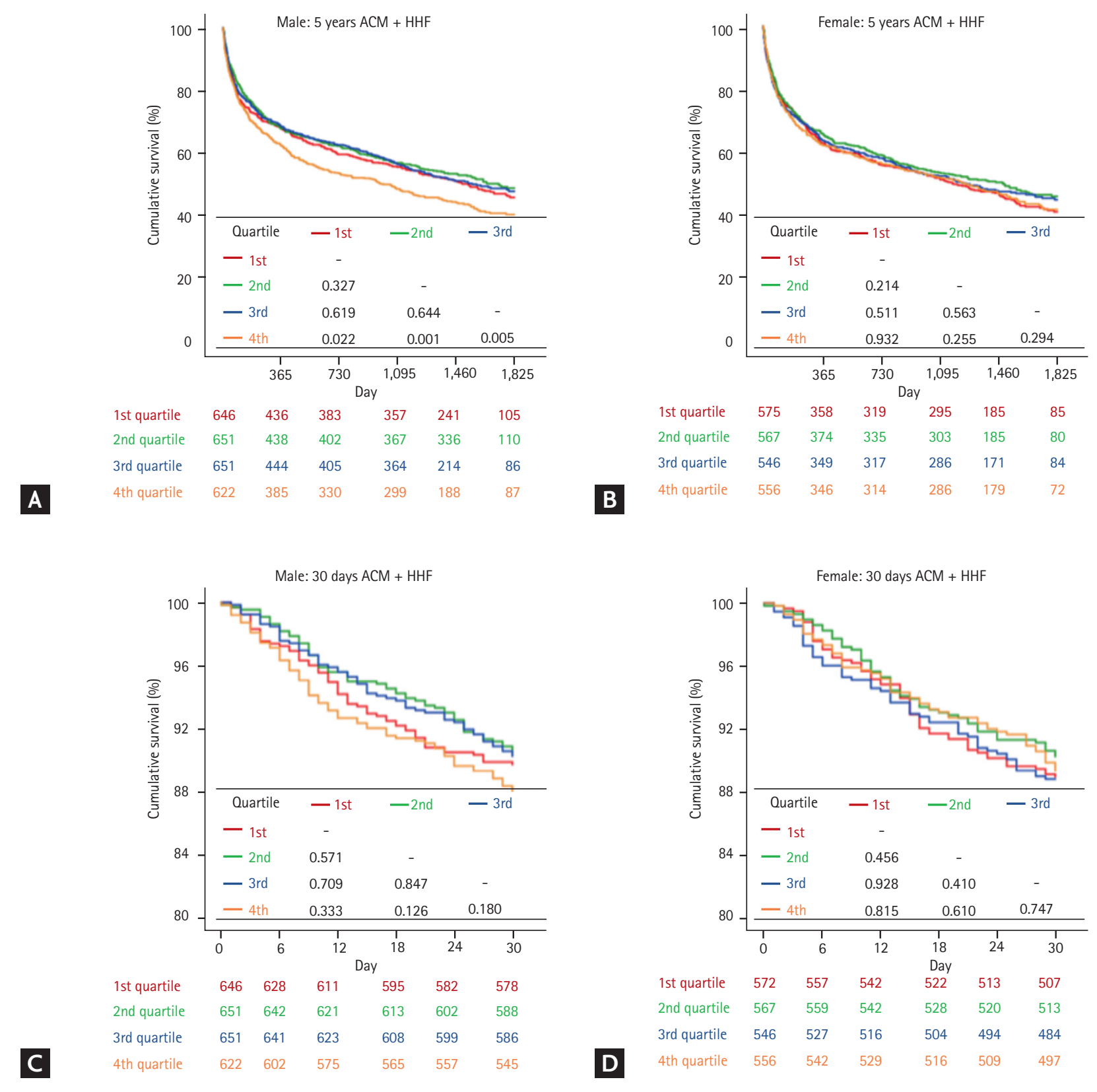

Supplementary Figure 1. Composite outcomes of all-cause mortality (ACM) and hospitalization due to heart failure (HHF) according to corrected QT (QTc) quartiles in acute heart failure patients. (A) Male: 5 years ACM + HHF. (B) Female: 5 years ACM + HHF. (C) Male: 30 days ACM + HHF. (D) Female: 30 days ACM + HHF. The Kaplan-Meier curves showed 5-year and 30 -day outcomes in male and female patients. 